\title{
Special issue: the International Conference on Soft Computing and Machine Intelligence
}

\author{
Suash Deb ${ }^{1,2} \cdot$ Thomas Hanne ${ }^{3} \cdot$ Ka-Chun Wong ${ }^{4}$ \\ Published online: 10 August 2018 \\ c) Springer-Verlag GmbH Germany, part of Springer Nature 2018
}

It is our privilege to present this Special Issue (SI) of the journal "Soft Computing" as guest editors. Before mentioning the papers of this SI, we would like to briefly present the background of the International Conference on Soft Computing and Machine Intelligence (ISCMI) series. Together with a new rise of artificial intelligence, the field of soft computing has further matured over the last decade regarding methodology, applications in various fields, and theoretical background leading to a vast amount of research results. ISCMI was established as a broad interdisciplinary forum where scientists and practitioners can present and discuss respective research works and applications addressing the progress of intelligent computing. Despite significant progress in computation power in many areas, good algorithms are still the main key to overcome limitations due to the complexity of problems or vagueness in the models or data.

ISCMI16 was the third conference in this series and was held from November 23 to 25, 2016, in Dubai. After a review of the conference submissions, 47 papers plus three keynote speeches were presented at the conference. From those papers, 16 were examined and shortlisted by experts for a possible inclusion in this SI after an extension of the paper by at least thirty percent. Ten of these 16 papers were

Thomas Hanne

thomas.hanne@fhnw.ch

Suash Deb

suashdeb@gmail.com

Ka-Chun Wong

kc.w@cityu.edu.hk

1 IT \& Educational Consultant, Ranchi, Jharkhand, India

2 Decision Sciences and Modelling Program, Victoria University, Melbourne, Australia

3 University of Applied Sciences and Arts Northwestern Switzerland, Olten, Switzerland

4 City University of Hong Kong, Kowloon Tong, Hong Kong SAR finally included in this SI after a regular review, as for every paper published in Soft Computing.

However, these papers represent a somewhat unbalanced distribution of key approaches in soft computing, i.e., artificial neural networks, fuzzy logic, and evolutionary computation. Five of the papers mainly or partially focus on swarm intelligence and evolutionary computation, while two use fuzzy approaches and one uses a neural network-based technique. In other papers, stronger focus is put on other approaches for clustering, or prediction, or even on the area of text mining. The contributions are elaborated in detail as follows:

In the paper by Yang et al. the past, present, and future of swarm intelligence (SI) are discussed. The paper provides a brief but up-to-date analysis of SI-based algorithms and their links with self-organization and various mathematical and qualitative characteristics and properties. Future research directions are discussed, including open questions from this research field. The paper by Grobler and Engelbrecht discusses two novel operators for dynamic particle swarm optimization, the arithmetic and the parent-centric headless chicken crossover operators. Seven variations of particle swarm optimization using related operators are investigated and evaluated in a range of benchmark problems with competitive performance results.

In the paper by Deb et al. the Elephant Search Algorithm (ESA) is applied to data clustering in the context of Kmeans. The hybrid approach suggests a good balance of local intensification and global exploration. Compared to classical clustering algorithms, the suggested approach shows superior results regarding clustering accuracy, also when applied to time-series clustering. In the other paper by Deb et al. a novel metaheuristic called Rhinoceros Search Algorithm (RSA) is suggested. The approach mimics several habitual characteristics of rhinoceros and simplifies search operations compared to the Elephant Search Algorithm. In some experiments, it is shown that the new approach outperforms several traditional metaheuristics on standard benchmark functions. Moreover, the new approach is adapted for the permutation flow shop 
scheduling problem and compared to particle swarm optimization, showing promising results again.

In the paper by Saha et al. a clustering problem is formulated in the context of gene and cancer classification using a fuzzy cluster ensemble approach in combination with multiobjective clustering techniques. Two optimization approaches, differential evolution and particle swarm optimization, are used to optimize two cluster quality measures, the Xie-Beni index and the FSym index. The potential of the new approach is shown in comparison with several stateof-the-art methods. In the paper by Herawan et al. a neural network approach based on multilayer perceptrons is used to predict the performance of a naturally aspirated spark ignition engine equipped with a waste heat recovery mechanism (WHRM). The results show good accuracy on training, test, and validation data. The paper by Hosni et al. deals with problems in software development effort estimation. Due to the difficulty of this problem, Ensemble Effort Estimation (EEE) is suggested to predict the software development effort by combining several single estimation techniques. Based on four well-known machine learning techniques, a heterogeneous ensemble approach is proposed and evaluated by investigating the impact of parameter values using two optimization techniques (grid search optimization and particle swarm optimization). It is shown that the approach leads to estimates that are more accurate. The paper by Datta and Mengel deals with the problem of deriving not too complex and better understandable rules from decision trees. This study tries to optimize the decision tree for the generation of rules specific to the infrequent class, which presents another challenge due to too few instances in the dataset. Two mining techniques, decision trees and association mining, are utilized together through ensemble learning in an adaptable fashion. The results show the generation of understandable rules with a better coverage of the infrequent class.

In the paper by Ismail et al. the results from a sentiment analysis of Twitter data are presented. Using machine learning based on a fuzzy thesaurus and sentiment replacement, accuracy results above $80 \%$ are obtained and a comparison of results with previous work shows an outperformance regarding results of other methods as reported in the literature. The paper by Mouriño-García et al. deals with the problem of classifying news data. A hybrid classifier is suggested to enrich the traditional bag-of-words representation of text data with concepts extracted from Wikipedia as background knowledge for the semantic analysis. The proposed approach is benchmarked with several other approaches, and the results show clear performance increases.

We hope that this SI will foster further research in the field of soft computing and machine intelligence and stimulate the development of new methods, theory, and applications. We are grateful to Prof. Antonio Di Nola and Prof. Vincenzo Loia, the Editor-in-Chief of this journal and the editor responsible for special issues, for giving us the opportunity to publish this SI, as well as for their further support during the preparation of the SI. We also wish to acknowledge the fast and thorough help provided by the editorial office and especially that of Mr. Anand Venugopal. We would also like to express our thanks to the reviewers for sending their valuable review inputs in time for improving the papers. Finally, we would like to thank the authors for their good cooperation and the patience during the review process of their manuscripts.

\section{Compliance with ethical standards}

Conflict of interest The authors declare that they have no conflict of interest in relation to this manuscript.

Publisher's Note Springer Nature remains neutral with regard to jurisdictional claims in published maps and institutional affiliations. 